\title{
Comprehensive Analysis of Antibiotic-induced Agranulocytosis Using the Japanese Adverse Drug Event Report Database
}

\author{
Yuki Asai ${ }^{1}$, Takanori Yamamoto ${ }^{1}$, and Yasuharu Abe $^{1}$ \\ ${ }^{1}$ National Hospital Organization Mie Chuo Medical Center
}

April 14, 2021

\begin{abstract}
Although infrequent, drug-induced agranulocytosis can be stimulated by antibiotics. Here, we analyzed the Japanese Adverse Drug Event Report database to identify profiles of antibiotic-induced agranulocytosis. Ten of 60 antibiotics showed signals for agranulocytosis; the reporting odds ratios (95\% confidence intervals) for ampicillin/sulbactam, amikacin, cefmetazole, cefozopran, clindamycin, ciprofloxacin, imipenem/cilastatin, kanamycin, teicoplanin, and vancomycin were 2.65 (1.79-3.80), 2.49 (1.91-4.34), 4.48 (2.27-6.92), 2.77 (1.88-3.95), 1.64 (1.04-2.47), 2.01 (1.40-2.82), 2.78 (2.11-3.60), 6.05 (2.16-13.7), 2.05 (1.31$3.07)$, and 3.54 (2.73-4.54), respectively. The median times-to-onset of agranulocytosis for ampicillin/sulbactam, cefmetazole, cefozopran, clindamycin, imipenem/cilastatin, kanamycin, teicoplanin, and vancomycin were 20, 6, 10, 16, 12, 3, 18, and 13 days, respectively. The $95 \%$ confidence intervals of the Weibull shape parameter $\beta$ for these antibiotics were over and excluded 1 , indicating that the antibiotics were the wear out failure type. These findings provided insights into the characteristics of antibiotic-induced agranulocytosis.
\end{abstract}

British Journal of Clinical Pharmacology

Article type : Short Reports

Article title

Comprehensive Analysis of Antibiotic-induced Agranulocytosis Using the Japanese Adverse Drug Event Report Database

Authors

Yuki Asai*, Takanori Yamamoto, Yasuharu Abe

Affiliation

Pharmacy, National Hospital Organization Mie Chuo Medical Center; 2158-5 Hisaimyojincho, Tsu, Mie, 514-1101 Japan

\section{*Corresponding author}

Yuki Asai, Ph.D., Pharmacy, National Hospital Organization Mie Chuo Medical Center; 21585 Hisaimyojincho, Tsu, Mie, 514-1101 Japan Tel: +81-59-259-1211, Fax: +81-59-256-2651, Email:yuki0715asai@gmail.com

Ethics approval and consent to participate

Ethics approval and consent to participate were not applicable to this study because we used publicly available voluntarily provided reports.

Running title 
Investigation of Antibiotic-induced Agranulocytosis

Keywords

antibiotics; drug-induced agranulocytosis; Japanese Adverse Drug Event Report; time-to-onset analysis; spontaneous reporting system; vancomycin

What is already known about this subject

Agranulocytosis can be caused by antibiotics, but it is a rare adverse event.

What this study adds

Ten antibiotics, including ampicillin/sulbactam, amikacin, cefmetazole, cefozopran, clindamycin, ciprofloxacin, imipenem/cilastatin, kanamycin, teicoplanin, and vancomycin, may induce agranulocytosis.

Eight antibiotics (excluding amikacin and ciprofloxacin) were a wear out failure type, the profiles of amikacin and ciprofloxacin were consistent with the random failure type.

Word count $1,548 / 2,000$ words

Table count 2

Figure count 1

\section{Abstract}

Although infrequent, drug-induced agranulocytosis can be stimulated by antibiotics. Here, we analyzed the Japanese Adverse Drug Event Report database to identify profiles of antibiotic-induced agranulocytosis. Ten of 60 antibiotics showed signals for agranulocytosis; the reporting odds ratios (95\% confidence intervals) for ampicillin/sulbactam, amikacin, cefmetazole, cefozopran, clindamycin, ciprofloxacin, imipenem/cilastatin, kanamycin, teicoplanin, and vancomycin were 2.65 (1.79-3.80), 2.49 (1.91-4.34), 4.48 (2.27-6.92), 2.77 (1.88$3.95), 1.64$ (1.04-2.47), 2.01 (1.40-2.82), 2.78 (2.11-3.60), 6.05 (2.16-13.7), 2.05 (1.31-3.07), and 3.54 (2.734.54), respectively. The median times-to-onset of agranulocytosis for ampicillin/sulbactam, cefmetazole, cefozopran, clindamycin, imipenem/cilastatin, kanamycin, teicoplanin, and vancomycin were 20, 6, 10, 16, $12,3,18$, and 13 days, respectively. The $95 \%$ confidence intervals of the Weibull shape parameter $\beta$ for these antibiotics were over and excluded 1 , indicating that the antibiotics were the wear out failure type. These findings provided insights into the characteristics of antibiotic-induced agranulocytosis.

\section{Introduction}

Neutropenia is characterized as a decrease in the absolute neutrophil count, categorized as mild (1000$1500 / \mu \mathrm{L})$, moderate $(500-1000 / \mu \mathrm{L})$, or severe $(<500 / \mu \mathrm{L})[1]$. The most severe form, called agranulocytosis, is a life-threating condition in which the patient becomes highly susceptible to infectious diseases and shows a mortality rate of 10-20\% [2]. Agranulocytosis can be caused by a wide range of medications, including clozapine [3] and antibiotics [1], and infection control becomes particularly difficult in patients with antibiotic-induced agranulocytosis. Furthermore, in patients who continue to have a fever, it is necessary to switch to another antibiotic and add concomitant treatment with granulocyte-colony-stimulating factor [4]. Accordingly, there is an urgent need to identify antibiotics that have a low risk of inducing agranulocytosis.

Although the mechanisms of antibiotic-induced agranulocytosis remain unknown, Weitzman and Stossel [5] speculated that antibiotic-induced agranulocytosis may be caused by opsonizing antineutrophil antibodies, i.e., an IgG or IgM immune-mediated hypersensitivity reaction. However, because agranulocytosis is a rare adverse event (AE) [6], it is difficult to collect a sufficient number of samples to conduct clinical studies. Recently, spontaneous reporting systems (SRSs) have been shown to be useful for detection of rare AEs and have been used as primary tools in postmarketing surveillance. In Japan, individual AEs are collected by healthcare workers at the Pharmaceuticals and Medical Devices Agency (PMDA). The Japanese Adverse Drug Event Report (JADER) database, which is available online, was established in April 2004 by the PMDA 
and reflects the realities of clinical practice [7]. Thus, data mining using the JADER database can be used as a screening tool for antibiotic-induced agranulocytosis.

In this study, we evaluated the expression profiles of antibiotic-induced agranulocytosis using the JADER database.

\section{Methods}

\section{Data source}

The JADER database, including data from April 2004 to January 2021, was searched from the PMDA website (https://www.info.pmda.go.jp/fukusayoudb/CsvDownload.jsp). The JADER database consisted of four data tables, as follows: 1) DEMO (patients' demographic information, including sex, age, and weight); 2) DRUG (drug name, causality, etc.); 3) REAC (AEs, outcomes, etc.); and 4) HIST (medical history, primary illness, etc.). We created relational database from the four data tables according to patient identification numbers using Microsoft Access 2019 (Microsoft, Redmond, WA, USA). Because duplicate reports in the SRS database may affect the evaluation from the relational database, we extracted only the latest of AE reports based on the DEMO file. Data with inaccurate dates were excluded.

Drugs and $A E$ names

The 60 antibiotics analyzed in the current study are shown in Table 1. We focused on agranulocytosis, the most life-threatening type of neutropenia. Agranulocytosis was defined using the preferred term (PT) code (10001507) according to the Medical Dictionary for Regulatory Activities version 23.1J.

\section{Data analysis}

We selected the reporting odds ratio (ROR) for the detection of signals associated with antibiotic-induced agranulocytosis. As shown Fig. 1, the ROR and 95\% confidence interval (95\% CI) were calculated from a two-by-two contingency table. Signal detection was defined as follows: ROR greater than 1, lower limit of the corresponding 95\% CI greater than 1, and case number greater than 2 [8]. Conversely, the condition of ROR less than 1 was considered no exposure-event association. The adjusted odds ratio was not calculated by multivariate analysis because of the small number of reports of agranulocytosis. Statical analysis was conducted with SPSS Statistics version 27 (IBM Japan, Tokyo, Japan).

The median of period until agranulocytosis onset from the time of first administration for each patient was evaluated using the Weibull shape parameter (WSP), which does not require a reference population [9]. The elapsed time from the first administration was calculated by subtracting the first administration date from the agranulocytosis expression date. Fifty cases were excluded because the date of first administration or onset of agranulocytosis was not available. The combination of WSP $\beta$ and $95 \%$ CI could be interpreted as follows: when $\beta$ was equal to 1 (random failure) and $95 \%$ CI of $\beta$ included the 1 , the hazard was estimated to be constant over time; if $\beta$ was greater than 1 (wear out failure) and the $95 \%$ CI (lower) of $\beta$ exceeded 1, the hazard was considered to increase over time; if $\beta$ was less than 1 (early failure) and the $95 \%$ CI (upper) of $\beta$ was less than 1 , the hazard was considered to increase at an early stage and then decrease. Time-to-onset analyses were performed using JMP version 15 software (SAS Institute, Cary, NC, USA).

\section{Results and Discussion}

We extracted 72,723 reports of suspected AEs related to antibiotic treatment among the 1,048,576 reports in the JADER database between April 2004 and January 2021. The number of reports of agranulocytosis was 3,221. As shown Table 1, 10 of 60 antibiotics were detected as signals; the RORs (95\% CIs) for ampicillin/sulbactam, amikacin, cefmetazole, cefozopran, clindamycin, ciprofloxacin, imipenem/cilastatin, kanamycin, teicoplanin, and vancomycin were 2.65 (1.79-3.80), 2.49 (1.91-4.34), 4.48 (2.27-6.92), 2.77 (1.88$3.95), 1.64$ (1.04-2.47), 2.01 (1.40-2.82), 2.78 (2.11-3.60), 6.05 (2.16-13.7), 2.05 (1.31-3.07), and $3.54(2.73-$ $4.54)$, respectively. Because neutrophils are produced in bone marrow, we speculated that antibiotics with high migration to bone marrow may have a high risk of inducing agranulocytosis before data mining. However, signals were not detected for antibiotics with high cerebrospinal fluid transferability, such as ceftriaxone 
[10] and cefotaxime [11], whereas teicoplanin [12], an antibiotic with poor cerebrospinal fluid transferability, was detected. Therefore, these findings suggested that the risk of agranulocytosis could not be clarified by analysis of migration to the bone marrow only. A retrospective study reported that the development of vancomycin-induced neutropenia was not associated with the dosage, trough concentration, or administration period [13]. Although other antibiotics also need to be investigated, antibiotic-induced agranulocytosis may not be a cumulative toxicity.

The incidence of neutropenia induced by vancomycin is approximately $2-18 \%$ [14, 15]. In contrast, Smith et al. reported that linezolid, an anti-methicillin-resistant Staphylococcus aureus (MRSA) agent, may be safe and effective for the treatment of febrile neutropenia [16]. In addition, carbapenems targeting Pseudomonas aeruginosa are empirically used for the treatment of febrile neutropenia [17], indicating that when using antibiotics with anti-Pseudomonas aeruginosa or anti-MRSA activity in the empirical treatment of high-risk patients, such as those with febrile neutropenia, it may be better to preferentially use antibiotics with the same spectrum for which no signal was detected in this study.

In the clinical setting, aminoglycosides are often administered with $\beta$-lactam antibiotics to induce synergistic effects $[18,19]$. Moreover, infective endocarditis caused by MRSA may be treated with vancomycin or teicoplanin in combination with aminoglycosides [20]. In the current study, because amikacin and kanamycin were detected as signals, we speculate that it may be better to use gentamicin as the aminoglycoside in combination therapy.

In recent reports of SRS data mining, WSP analysis has been performed to evaluate the time-to-onset data for target AEs [9]. For the time-to-onset analysis, we extracted 292 cases for the 10 antibiotics with signal detection (Table 2). The median durations (interquartile ranges) of agranulocytosis onset following treatment with amikacin, cefmetazole, ciprofloxacin, and kanamycin were within 1 week of the first treatment. The difference in the onset time may have been related to the variations in pharmacokinetics of each antibiotic and the onset mechanism of agranulocytosis; however, the details are unknown, and further research is needed. Although the $95 \%$ CIs of WSP $\beta$ for eight antibiotics (excluding amikacin and ciprofloxacin) were over and excluded 1, indicating a wear out failure type, the profiles of amikacin and ciprofloxacin were consistent with the random failure type (Table 2). Accordingly, time-to-onset analysis using WSP is likely to be a useful tool for determination of the specific safety monitoring period for agranulocytosis.

Data mining using the JADER database has some limitations that should be considered because the JADER database has several biases. First, because the JADER database does not contain information for control patients, the intensity of RORs cannot be quantified and compared among antibiotics. Second, there are also some reporting biases, such as under-reporting and lack of data. Third, the number of reports was small and may have been underestimated because the PT codes 10018687 (granulocytopenia) and 10018681 (granulocyte count decreased) were not included in the current study. Fourth, because agranulocytosis may be induced by severe infections [21], the agranulocytosis reported by SRS may not be caused by administration of antibiotics.

In conclusion, this comprehensive analysis was the first report evaluating the incidences of antibiotic-induced agranulocytosis. Despite the various limitations of using the JADER database, we identified 10 antibiotics that may be associated with high risk of agranulocytosis, suggesting that absolute neutrophil counts in patients taking these drugs should be monitored carefully in the clinical setting. Finally, further clinical studies are needed to verify the mechanisms through which ampicillin/sulbactam, amikacin, cefmetazole, cefozopran, clindamycin, ciprofloxacin, imipenem/cilastatin, kanamycin, teicoplanin, and vancomycin may induce agranulocytosis.

\section{Acknowledgments}

We would like to thank Editage (www.editage.com) for English language editing.

Conflict of interest statement

The authors have declared that there is no conflict of interest. 
Funding information

Non

Data availability statement

The data that support the findings of this study are available from the corresponding author upon reasonable request.

Authorship

Concept and study design: Asai, Abe

Assembly and interpretation of data: Asai, Yamamoto, Abe

Manuscript writing: Asai

Final approval of manuscript: Asai, Yamamoto, Abe

Accountable for all aspects of the work: Asai, Yamamoto, Abe

ORCID

Yuki Asai: https://orcid.org/0000-0002-1933-8592

\section{References}

1. Boxer LA. How to approach neutropenia. Hematology Am Soc Hematol Educ Program, 2012; 2012: 174-182.

2. Andrès E, Maloisel F, Kurtz JE, Kaltenbach G, Alt M, Weber JC, Sibilia J, Schlienger JL, Blicklé JF, Brogard JM, Dufour P. Modern management of non-chemotherapy drug-induced agranulocytosis: a monocentric cohort study of 90 cases and review of the literature. Eur J Intern Med. 2002; 13: 324-328.

3. Mijovic A, MacCabe JH. Clozapine-induced agranulocytosis. Ann Hematol. 2020; 99: 2477-2482.

4. Genchanok Y, Tolu SS, Wang H, Arora S. Agranulocytosis from Outpatient Antimicrobial Treatment with Ceftriaxone: A Case Report. Perm J. 2019; 23: 18-190.

5. Weitzman SA, Stossel TP. Drug-induced immunological neutropenia. Lancet. 1978; 1: 1068-1072.

6. Olaison L, Belin L, Hogevik H, Alestig K. Incidence of beta-lactam-induced delayed hypersensitivity and neutropenia during treatment of infective endocarditis. Arch Intern Med. 1999; 159: 607-615.

7. Noguchi Y, Ueno A, Otsubo M, Katsuno H, Sugita I, Kanematsu Y, Yoshida A, Esaki H, Tachi T, Teramachi H. A simple method for exploring adverse drug events in patients with different primary diseases using spontaneous reporting system. BMC Bioinformatics. 2018; 19: 124.

8. Hashiguchi M, Imai S, Uehara K, Maruyama J, Shimizu M, Mochizuki M. Factors Affecting the Timing of Signal Detection of Adverse Drug Reactions. PLoS One. 2015; 10: e0144263.

9. Sauzet O, Carvajal A, Escudero A, Molokhia M, Cornelius VR. Illustration of the weibull shape parameter signal detection tool using electronic healthcare record data. Drug Saf. 2013; 36: 995-1006.

10. Kotani A, Hirai J, Hamada Y, Fujita J, Hakamata H. Determination of ceftriaxone concentration in human cerebrospinal fluid by high-performance liquid chromatography with UV detection. J Chromatogr B Analyt Technol Biomed Life Sci. 2019; 1124: 161-164.

11. Chen XK, Shi HY, Leroux S, Xu HY, Zhou Y, Zheng Y, Huang X, Li Y, Jacqz-Aigrain E, Zhao W. Penetration of Cefotaxime into Cerebrospinal Fluid in Neonates and Young Infants. Antimicrob Agents Chemother. 2018; 62: e02448-17.

12. Wilson AP. Clinical pharmacokinetics of teicoplanin. Clin Pharmacokinet. 2000; 39: 167-183.

13. Pai MP, Mercier RC, Koster SA. Epidemiology of vancomycin-induced neutropenia in patients receiving home intravenous infusion therapy. Ann Pharmacother. 2006; 40: 224-228.

14. Farber BF, Moellering RC Jr. Retrospective study of the toxicity of preparations of vancomycin from 1974 to 1981. Antimicrob Agents Chemother. 1983; 23: 138-141.

15. Morris A, Ward C. High incidence of vancomycin-associated leucopenia and neutropenia in a cardiothoracic surgical unit. J Infect. 1991; 22: 217-223. 
16. Smith PF, Birmingham MC, Noskin GA, Meagher AK, Forrest A, Rayner CR, Schentag JJ. Safety, efficacy and pharmacokinetics of linezolid for treatment of resistant Gram-positive infections in cancer patients with neutropenia. Ann Oncol. 2003; 14: 795-801.

17. Khoo AL, Zhao YJ, Teng M, Ying D, Jin J, Chee YL, Poon LM, Lim SE, Koh LP, Chng WJ, Lim BP, Hsu LY, Chai LYA. Evaluation of a risk-guided strategy for empirical carbapenem use in febrile neutropenia. Int J Antimicrob Agents. 2018; 52: 350-357.

18. Kurtz TO, Winston DJ, Bruckner DA, Martin WJ. Comparative in vitro synergistic activity of new beta-lactam antimicrobial agents and amikacin against Pseudomonas aeruginosa and Serratia marcescens. Antimicrob Agents Chemother. 1981; 20: 139-243.

19. Scudamore RA, Goldner M. Penetration of the outer membrane of Pseudomonas aeruginosa by synergistic combinations of beta-lactam and aminoglycoside antibiotics. Antimicrob Agents Chemother. 1982; 21: 1007-1010.

20. Baddour LM, Wilson WR, Bayer AS, Fowler VG Jr, Tleyjeh IM, Rybak MJ, Barsic B, Lockhart PB, Gewitz MH, Levison ME, Bolger AF, Steckelberg JM, Baltimore RS, Fink AM, O'Gara P, Taubert KA; American Heart Association Committee on Rheumatic Fever, Endocarditis, and Kawasaki Disease of the Council on Cardiovascular Disease in the Young, Council on Clinical Cardiology, Council on Cardiovascular Surgery and Anesthesia, and Stroke Council. Infective Endocarditis in Adults: Diagnosis, Antimicrobial Therapy, and Management of Complications: A Scientific Statement for Healthcare Professionals From the American Heart Association. Circulation. 2015; 132: 1435-1486.

21. Shi R, Zhou Q, Fang R, Xiong X, Wang Q. Severe acute pancreatitis with blood infection by Candida glabrata complicated severe agranulocytosis: a case report. BMC Infect Dis. 2018; 18: 706.

\section{Figure Legends}

Fig. 1. Two-by-two contingency table for the calculation of reporting odds ratios.

\section{Hosted file}

Table.pdf available at https://authorea.com/users/407713/articles/517953-comprehensiveanalysis-of-antibiotic-induced-agranulocytosis-using-the-japanese-adverse-drug-eventreport-database

\section{Hosted file}

Fig.1.pptx available at https://authorea.com/users/407713/articles/517953-comprehensiveanalysis-of-antibiotic-induced-agranulocytosis-using-the-japanese-adverse-drug-eventreport-database 